\title{
Does a physiology-based interpretation of CTG allow to dispense with second-line methods? A cross-sectional online survey
}

\author{
Manon Marquet ${ }^{1}$, Julie Blanc ${ }^{1}$, Claude D'Ercole ${ }^{2}$, Xavier Carcopino ${ }^{1}$, Florence Bretelle ${ }^{3}$, \\ and Antoine Netter ${ }^{1}$ \\ ${ }^{1} \mathrm{AP}-\mathrm{HM}$ \\ ${ }^{2}$ Assistance Publique - Hôpitaux de Marseille \\ ${ }^{3}$ Hopital Nord, AP-HM, Aix Marseille University
}

January 6, 2022

\begin{abstract}
Objective: To determine the influence of a training course in physiology-based interpretation of cardiotocography (CTG) on professional practices. Design: A cross-sectional study. Setting: A national online survey. Population: Fifty-seven French obstetricians Methods: The participants were divided into two groups: the training group (obstetricians who had already participated in a training course in physiology-based interpretation of CTG) and the control group. Ten medical records of patients who had abnormal CTG tracings and underwent a foetal scalp blood sampling $(\mathrm{pH})$ during labour were presented to the participants. They were given three choices: (i) use a second-line method, (ii) continue labour without using a second-line method, or (iii) perform a caesarean section. Main outcome measures: Median number of decisions to use second-line methods. Results: Forty participants were included in the training group and 17 in the control group. The median number of recourses to a second-line method was significantly inferior for the training group (4 [2-6] second-line methods on 10 records) than for the control group $(6$ [4-7]; $\mathrm{p}=0.040)$. Regarding the 4 records for which a caesarean section was the real outcome, the median number of decisions of continuing labour was significantly superior in the training group than in the control group (1 [0-1] vs. $0[0-1] ; \mathrm{p}=0.032)$. Conclusions: Participation in a training course in physiology-based interpretation of CTG could be associated with a less frequent use of second-line methods. Additional studies are required to determine whether this change in attitude is safe for the foetal well-being.
\end{abstract}

\section{Introduction}

Electronic foetal monitoring was invented in the 1960s, and cardiotocography (CTG) has since become the primary means of monitoring foetal well-being during labour in developed countries ${ }^{1,2}$. CTG interpretation has an excellent negative predictive value but carries the drawback of a mediocre positive predictive value ${ }^{3}$. Thus, the interpretation of an abnormal CTG is challenging, and misinterpretations are frequent with the implicit risk of performing excessive foetal extractions or conversely, to wrongly abstain from an intervention that would have been necessary to preserve foetal well-being ${ }^{4,5}$. CTG is therefore an imperfect tool to be used alone to make decisions with potentially serious consequences, often in emergency situations. In this context, second-line methods have found their use in cases of abnormal CTG and they allow us to differentiate abnormalities that reflect foetal acidosis from those that do not have an impact on foetal well-being ${ }^{6}$.

This rationale has recently been challenged. Some authors have suggested that foetal monitoring does not inherently have a low positive predictive value and that its diagnostic performance could be improved by a paradigm shift in its interpretation ${ }^{7}$. Their claim is that CTG should not be interpreted on a pattern recognition basis but with a pathophysiological approach instead ${ }^{8-10}$. Indeed, traditionally, the interpretation of the CTG is taught by learning distinct criteria of concern (baseline heart rate, absence of accelerations, 
short-term variability, presence of decelerations) and by the recognition of different patterns of decelerations (early, variable or late; typical or atypical) ${ }^{11}$. The new paradigm is based on pathophysiology studies, especially in animals, and promotes the teaching of explanations of the foetal response to various phenomena occurring during labour ${ }^{8,12}$. One of the main purposes of this method is to differentiate between tracings that correspond to a physiological adaptation of the foetus and those that have a decompensatory meaning and require immediate foetal extraction. The underlying implicit goal is to sufficiently improve the positive predictive value of the CTG interpretation to dispense with second-line methods ${ }^{13-15}$. Since 2017 and the publication of the "Handbook of CTG Interpretation: From Patterns to Physiology", this method of interpretation has been widely promoted, particularly through specific training courses ${ }^{8}$. However, there are no existing studies to determine the value of these teachings on clinical decision-making.

The objective of this study was to evaluate the impact of specific training in CTG physiology-based interpretation on professional attitudes towards the use of second-line methods.

\section{Methods}

Study design and participants

We conducted a national cross-sectional study based on an online survey. French obstetricians were invited to participate during congresses and through national networks. Participants could be licenced obstetricians or specialized residents in their fourth year or higher and were required to have had regular practice in obstetrics in the previous year. This study was approved by the local ethics committee (Aix-Marseille University 2021-04-08-06).

Questionnaire

The questionnaire was divided into two parts:

The first part included questions about the participant: level of experience, place of work, frequency of use of second-line methods (frequent or infrequent), participation in specific training in physiology-based CTG interpretation and the type of training.

The second part focused on clinical cases. Ten medical records of patients who delivered in our unit and for whom foetal scalp blood sampling $(\mathrm{pH})$ was performed during labour were presented with the following information: medical history, notable events during pregnancy, detailed description of the labour with a partograph and CTG. All presented information was anterior to the decision to perform foetal scalp blood sampling: information about what happened after this decision was hidden from the participants on the partograph and the CTG. For each medical record, the participants were given three options: (i) perform a second-line method, (ii) continue labour without performing a second-line method, or (iii) perform a caesarean section without performing a second-line method.

After completion of the questionnaire, the participants received an email in which the real outcomes (foetal scalp blood sampling, mode of delivery, neonatal status) were presented for each medical record. They could not modify their answers after their completion of the questionnaire.

Selection of medical records

Ten medical records of term pregnant women who delivered in our unit between 2019 and 2020 and presented CTG abnormalities for whom a second-line method was used were carefully selected among forty records. Four reviewers were involved in this selection process and had access to the entire record (before and after foetal scalp blood sampling, including the neonatal status). The four reviewers were experienced obstetricians, including one expert in the court.

They were given the same instructions:

For patients for whom a caesarean section was performed, the record could be retained only if the foetal scalp blood sampling $(\mathrm{pH})$ was less than 7.20 and if the reviewers deemed that caesarean section was the only reasonable decision to preserve foetal well-being. The record could not be retained if one or more 
reviewers deemed that another reasonable solution existed to avoid or delay a caesarean section (e.g., begin expulsive efforts, modify oxytocin administration). According to these criteria, four records of patients for whom a caesarean section was performed after foetal scalp blood sampling were retained and presented to the participants.

For patients who had a vaginal delivery, the record could be retained only if the foetal scalp blood sampling $(\mathrm{pH})$ was greater than 7.20 , if the delivery occurred at least one hour after the foetal scalp blood sampling and if the reviewers deemed that continuing the labour had been a reasonable decision based on the neonatal status. According to these criteria, six records were selected.

The four reviewers had to agree that a medical record met the required criteria for it to be selected. In any case of disagreement among the reviewers, the medical record was not retained for presentation. Their judgement was based on the entire medical record, including the neonatal status after delivery.

Groups and endpoints

The participants were divided into two groups: the training group (obstetricians who participated in a training course in physiology-based interpretation of CTG) and the control group (no participation in such a course declared).

The primary endpoint was the difference between groups in the median number of second-line methods that the participants would have performed.

The secondary endpoints were the median number of decisions to continue labour without a second-line method when the real outcome was a caesarean section and the median number of decisions to perform a caesarean section when the real outcome was a vaginal delivery.

Statistical analyses

The number of participants was calculated to detect an arbitrary median difference between groups of one recourse to a second-line method among the ten presented records. Given a two-sided test with $90 \%$ statistical power and 95\% confidence interval (CI), 44 participants needed to be included. Based on this number and with the assumption that 10 to 15 more obstetricians would agree to participate after each monthly reminder, we decided to allow access to the online questionnaire for four months (July to October 2020).

Professional characteristics of the participants and attitudes towards the presented cases were compared between the two groups. Qualitative variables are presented as counts (percentages) and were compared between groups using the $\chi^{2}$ test or Fisher's exact test when appropriate. Continuous variables are presented as the median (interquartile range [IQR]) and were compared using Student's t-test or the Mann-Whitney $\mathrm{U}$ test when appropriate.

All of the statistical analyses were two-tailed, and the results were taken to be statistically significant when $\mathrm{P}$ values $<0.05$ were obtained. The analyses were performed using IBM SPSS Statistics version 20.0 (IBM Corporation, Armonk, New York, US).

\section{Results}

Characteristics of the participants

The participants' characteristics are reported in Table 1 . Fifty-seven obstetricians answered the questionnaire: 40 had already participated in specific training in physiology-based CTG interpretation and were included in the training group, and 17 participants were included in the control group. The level of experience was the same in both groups $(\mathrm{p}=0.623)$. The majority of participants were residents (in their fourth year or higher) and worked in a university hospital. Participants in the training group either participated in a two-day master class $(27.5 \%)$, had a shorter training course $(72.5 \%)$ or read a book on physiology-based CTG interpretation (25.0\%). Most of the participants in the training group declared using this teaching in their daily practice $(95.0 \%)$. 


\section{Attitudes towards clinical cases}

The participants' decisions towards the clinical cases are summarized inTable 2 . Among the ten medical records, the median number of recourses to a second-line method was significantly inferior for the training group (4 [2-6] second-line methods on 10 records) than for the control group $(6[4-7] ; \mathrm{p}=0.040)$. The median number of decisions to continue labour without using a second-line method was significantly superior in the training group than in the control group $(3[2-5]$ vs. $2[1-3] ; p=0.015)$. The median number of decisions to perform a caesarean section without using a second-line method was similar between groups (2 [1-4] vs. 2 $[1-3.5] ; \mathrm{p}=0.788)$.

Among the 4 records for which a caesarean section was in fact performed after the foetal scalp blood sampling, the median number of decisions of continuing labour without using a second-line method would have been significantly superior in the training group than in the control group (1 [0-1] decision of continuing labour on 4 records vs. $0[0-1] ; \mathrm{p}=0.032$ ). Among the 6 records for which the patient actually delivered vaginally after the result of the foetal scalp blood sampling, the median number of decisions of performing a caesarean section without using a second-line method would have been the same between groups $(0$ [0-2] caesarean sections on 6 records vs. $1[0-2] ; \mathrm{p}=0.641)$. Among 4 records for which the result of foetal scalp blood sampling $(\mathrm{pH})$ was superior to 7.30 , the median number of decisions to perform a second-line method was the same for both groups $(0[0-1]$ vs. $0[0-1] ; \mathrm{p}=0.921)$.

Examples of medical records

The description of the medical records with the answers from the participants can be found in Table $\mathbf{3}$. We also provide a detailed description of the two medical records for which the rates of inconsistent answers with regard to the real outcome were the greatest (Figure $\mathbf{1}$ ):

Medical Record \#7: A 24-year-old woman, gravida 1, para 0, presented at 38 weeks of gestation with oligohydramnios and decreased active foetal movements in the setting of gestational diabetes with foetal macrosomia (estimated foetal weight above the 99th percentile). Induction of labour was achieved with oxytocin under epidural anaesthesia. CTG abnormalities appeared at $9 \mathrm{~cm}$ of cervical dilatation and led to foetal scalp blood sampling (Figure 1a ). The result was 7.27, and the obstetrician decided to continue labour with iterative foetal scalp blood sampling. One hour after the first sampling, the patient vaginally delivered a 3,800 gram newborn boy. The Apgar scores were 10, 10, and 10 at 1, 5 and 10 minutes, respectively, and the umbilical cord $\mathrm{pH}$ was 7.17 .

Regarding this medical record, $42 \%$ of participants would have decided to perform a caesarean section without using a second-line method (38\% of the participants in the training group and $53 \%$ of the participants in the control group). Only 3 participants (7\%) from the training group would have decided to continue labour without using a second-line method, and no participant from the control group would have decided to continue labour.

Medical Record \#9: A 38-year-old woman, gravida 1, para 0, presented in labour at 39 gestational weeks in the setting of gestational diabetes without foetal macrosomia. CTG abnormalities appeared at $6 \mathrm{~cm}$ of cervical dilatation and led to foetal scalp blood sampling (Figure 1b ). The result was 7.16, and the obstetrician decided to perform a caesarean section. The newborn boy weighed 2605 grams and had Apgar scores of 8, 9, and 9 at 1, 5 and 10 minutes, respectively, with an umbilical cord pH of 7.14. He was admitted to the neonatal intensive care unit for respiratory distress and required continuous positive airway pressure for 24 hours.

Regarding this medical record, $47 \%$ of participants would have decided to continue labour without using a second-line method (55\% of the participants in the training group and $29 \%$ of participants in the control group). Only two participants (5\%) from the training group would have chosen to perform a caesarean section without using a second-line method, and one from the control group (6\%) would have chosen to perform a caesarean section.

\section{Discussion}




\section{Main Findings}

This study is the first to evaluate the influence of specific training in physiology-based CTG interpretation on professional attitudes towards recourse to second-line methods. Our results suggest that obstetricians who underwent this type of training would less often decide to use second-line methods when confronted with CTG abnormalities. This advantage would come at the cost of more often deciding to continue labour in situations that would have required a caesarean section to preserve neonatal well-being. Conversely, the obstetricians who did not undergo this training did not seem to opt more often for a caesarean section when the patient actually delivered vaginally.

Strengths and Limitations

The main strength of this study was the careful selection of medical records. Ten medical records were selected among forty others by four reviewers, including one expert in court. Clear instructions were given beforehand to the reviewers to only retain records whose outcome was unequivocal. In other words, the four reviewers had to agree that the actual mode of delivery (either a caesarean section or a vaginal delivery) was the best possible outcome in the clinical context with knowledge of the entire medical record, including the results of the foetal scalp blood sampling and the neonatal status. This rigorous selection process legitimises the claim that choosing an alternative mode of delivery without using a second-line method would at least result in a nonoptimal outcome for the patient or her newborn. The exhaustive and careful presentation of all the information necessary for the participants to make a decision should also be emphasized. Real CTGs were presented either entirely since the beginning of labour or partially since before the beginning of CTG abnormalities (when the tracings exceeded three hours). Hence, this online questionnaire allowed an original simulation of real clinical situations, and we believe that it is a reliable evaluation of the participants' professional attitudes.

However, several considerations mitigate the conclusions that can be drawn from the results of this study. Despite the aforementioned precautions, it is impossible to assert with absolute certainty the outcome of an alternative scenario. Although we consider it unlikely, it is not formally impossible that among the 4 records that resulted in a caesarean section, one or more records could have ended differently. Several participants also pointed out that reducing the choice to three options was too restrictive and did not give a fair representation of reality. In particular, it is possible that some participants would have opted to continue labour only for some time before ultimately performing a caesarean section or a second-line method. Similarly, some participants suggested that other options were available, such as positioning the patient differently or stopping oxytocic administration. As stated before, although four reviewers deemed that the actual outcome was the best possible option, alternative scenarios cannot formally be excluded.

In addition, there is no way of verifying that the participants would have actually made the stated decision in a real-life situation. It is possible that the context of the anonymous questionnaire, devoid of any stress related to the consequences and presented as a test, encouraged participants to take more risks in their decision-making. It is also worth mentioning that the majority of the participants of this online survey were residents. Although we only included residents in their fourth year or higher who are ordinarily already selfreliant in obstetrical decision-making, we can hardly extrapolate the results to more experienced physicians.

Furthermore, all modes of training for the CTG physiology-based interpretation were placed on an equal footing in the training group. The number of participants was insufficient to provide subgroup analysis for each type of training. Although the vast majority of the participants from the training group declared that they used the precepts of CTG physiology-based interpretation in their daily practice, it is possible that our results are biased by the insufficient training of some participants.

\section{Interpretation}

The underlying goal of physiology-based CTG interpretation is to dispense with second-line methods ${ }^{14,15}$. In a 2014 insightful review, Edwin Chandraharan pointed out the absence of evidence supporting foetal scalp blood sampling during labour ${ }^{13}$. This critique was brilliantly enriched with a detailed list of pathophysio- 
logical inconsistencies associated with the use of this test; in particular, a sample of blood from nonessential peripheral tissue (foetal scalp) is being used to assess the acid-base status of essential central organs. His conclusion was that foetal scalp blood sampling was an outdated practice and that it should no longer be recommended. This review provoked a debate among various authors whose responses were just as interesting as the original article, essentially discussing the pathophysiological basis of foetal scalp blood sampling ${ }^{16-18}$. These expert debates are enlightening, but no pathophysiological analysis can ever replace the evidence provided by a well-conducted clinical study. Since 2014, despite this article, which should have encouraged research on this matter, little has been done to demonstrate the usefulness of second-line methods, which are still widely used despite this lack of evidence.

The same reasoning could easily be applied to physiology-based CTG interpretation training. There is no doubt that pathophysiological teaching leads to a deeper understanding of medical phenomena and should be encouraged. Recent studies showed that training programmes based on foetal physiology led to a long-term improvement in CTG interpretation and to homogenization of obstetrical decisions in simulated situations ${ }^{19,20}$. However, there is a strong need for clinical evidence regarding the teaching of this method before totally discouraging second-line methods. As suggested by our results, overconfidence in the interpretation of CTG could lead to wrong decisions and it should therefore be taught with humility and by stressing the importance of being cautious when in doubt. The present article is up to date and, to our knowledge, is the only attempt to analyse the effect of physiology-based CTG interpretation training on obstetrical practice. Although our work is clearly insufficient on its own to substantiate the benefits or shortcomings of this teaching, it does have the merits of raising the issue of the lack of evidence and should encourage further research.

Conclusions

Our results suggest that participation in a training course in physiology-based interpretation of CTG could be associated with a less frequent use of second-line methods at the cost of more frequently continuing labour with the risk compromising foetal and maternal well-being. Neither this study nor the current state of the literature allows us to recommend abandoning second-line methods in favour of an enhanced CTG interpretation. The same methodology could be used on a larger scale to confirm or refute these results.

\section{Disclosure of Interests}

The authors declare no conflict of interest related to this study.

\section{Contribution to Authorship}

M.M. and A.N. developed the original design.

A.N. and J.B. performed data analysis and interpretation.

A.N. and M.M. wrote the first draft of the report.

All authors contributed to the writing of the final manuscript.

\section{References}

1. Hon EH, Khazin AF. Observations on fetal heart rate and fetal biochemistry: I. Base deficit. American Journal of Obstetrics and Gynecology [Internet]. 1969 Nov 1 [cited 2021 Jun 8];105(5):721-9. Available from: https://www.sciencedirect.com/science/article/pii/0002937869900088

2. Ayres-de-Campos D. Electronic fetal monitoring or cardiotocography, 50 years later: what's in a name? American Journal of Obstetrics and Gynecology [Internet]. 2018 Jun [cited 2021 Jun 8];218(6):545-6. Available from: https://linkinghub.elsevier.com/retrieve/pii/S0002937818302175

3. Fleischer A, Schulman H, Jagani N, Mitchell J, Randolph G. The development of fetal acidosis in the presence of an abnormal fetal heart rate tracing. I. The average for gestational age fetus. Am J Obstet Gynecol. 1982 Sep 1;144(1):55-60. 
4. Nelson KB, Dambrosia JM, Ting TY, Grether JK. Uncertain value of electronic fetal monitoring in predicting cerebral palsy. N Engl J Med. 1996 Mar 7;334(10):613-8.

5. Parer JT, King T. Fetal heart rate monitoring: is it salvageable? Am J Obstet Gynecol. 2000 Apr;182(4):982-7.

6. Haverkamp AD, Orleans M, Langendoerfer S, McFee J, Murphy J, Thompson HE. A controlled trial of the differential effects of intrapartum fetal monitoring. Am J Obstet Gynecol. 1979 Jun 15;134(4):399-412.

7. Ugwumadu A. Are we (mis)guided by current guidelines on intrapartum fetal heart rate monitoring? Case for a more physiological approach to interpretation. BJOG. 2014 Aug;121(9):1063-70.

8. Chandraharan E. Handbook of CTG Interpretation: From Patterns to Physiology. Cambridge University Press; 2017. 257 p.

9. Turner JM, Mitchell MD, Kumar SS. The physiology of intrapartum fetal compromise at term. Am J Obstet Gynecol. 2020 Jan;222(1):17-26.

10. Lear CA, Westgate JA, Ugwumadu A, Nijhuis JG, Stone PR, Georgieva A, et al. Understanding Fetal Heart Rate Patterns That May Predict Antenatal and Intrapartum Neural Injury. Semin Pediatr Neurol. 2018 Dec;28:3-16.

11. Ingemarsson I. Fetal Heart Rate Monitoring. Oxford ; New York: Oxford University Press; 1993. 339 p.

12. Lear CA, Kasai M, Booth LC, Drury PP, Davidson JO, Maeda Y, et al. Peripheral chemoreflex control of fetal heart rate decelerations overwhelms the baroreflex during brief umbilical cord occlusions in fetal sheep. J Physiol. 2020 Oct;598(20):4523-36.

13. Chandraharan E. Fetal scalp blood sampling during labour: is it a useful diagnostic test or a historical test that no longer has a place in modern clinical obstetrics? BJOG: An International Journal of Obstetrics \& Gynaecology [Internet]. 2014 [cited 2021 May 30];121(9):1056-62. Available from: https://obgyn.onlinelibrary.wiley.com/doi/abs/10.1111/1471-0528.12614

14. Chandraharan E. Should national guidelines continue to recommend fetal scalp blood sampling during labor? J Matern Fetal Neonatal Med. 2016 Nov;29(22):3682-5.

15. Chandraharan E. Fetal scalp blood sampling should be abandoned: FOR: FBS does not fulfil the principle of first do no harm. BJOG. 2016 Oct;123(11):1770.

16. Steer PJ. Fetal scalp blood sampling during labour: is it a useful diagnostic test or a historical test which has no longer a place in modern clinical obstetrics. BJOG. 2014 Dec;121(13):1749-50.

17. Nzewi C. Fetal scalp blood sampling during labour: is it a useful diagnostic test or a historical test that no longer has a place in modern clinical obstetrics? BJOG. 2014 Dec;121(13):1753.

18. Sholapurkar SL. Re: Fetal scalp blood sampling during labour: is it a useful diagnostic test or a historical test that no longer has a place in modern clinical obstetrics [corrected]? BJOG. 2014 Dec;121(13):1750.

19. Dupuis H, Ghesquière L, Pierache A, Subtil D, Houfflin-Debarge V, Garabedian C. Evaluation and impact of fetal physiology training on fetal heart rate analysis. Journal of Gynecology Obstetrics and Human Reproduction [Internet]. 2021 Dec 1 [cited 2021 Dec 21];50(10):102185. Available from: https://www.sciencedirect.com/science/article/pii/S2468784721001239

20. Zhu L-A, Blanc J, Heckenroth H, Peyronel C, Graesslin B, Marcot M, et al. Fetal physiology cardiotocography training, a regional evaluation. J Gynecol Obstet Hum Reprod. 2021 Jun;50(6):102039.

Tables and Figures

Table 1: Characteristics of the participants

Table 2: Comparison of attitudes towards clinical cases between the two groups. 
Table 3: Description of the medical records and detailed answers from the participants.

Figure 1: Examples of medical records. Left side: Partograph; Right side: Cardiotocograph. 1A: Medical Record \#7 before foetal scalp blood sampling. The result was 7.27 , and the obstetrician decided to continue labour with iterative foetal scalp blood sampling. The patient delivered vaginally.

1B: Medical Record \#9 before the foetal scalp blood sampling. The result was 7.16 , and the obstetrician decided to perform a caesarean section. The newborn was admitted to the neonatal intensive care unit for respiratory distress and required continuous positive airway pressure for 24 hours.

\section{Hosted file}

Table 1.docx available at https://authorea.com/users/454213/articles/551895-does-aphysiology-based-interpretation-of-ctg-allow-to-dispense-with-second-line-methods-across-sectional-online-survey

Hosted file

Table 2.docx available at https://authorea.com/users/454213/articles/551895-does-aphysiology-based-interpretation-of-ctg-allow-to-dispense-with-second-line-methods-across-sectional-online-survey

\section{Hosted file}

Table 3.docx available at https://authorea.com/users/454213/articles/551895-does-aphysiology-based-interpretation-of-ctg-allow-to-dispense-with-second-line-methods-across-sectional-online-survey

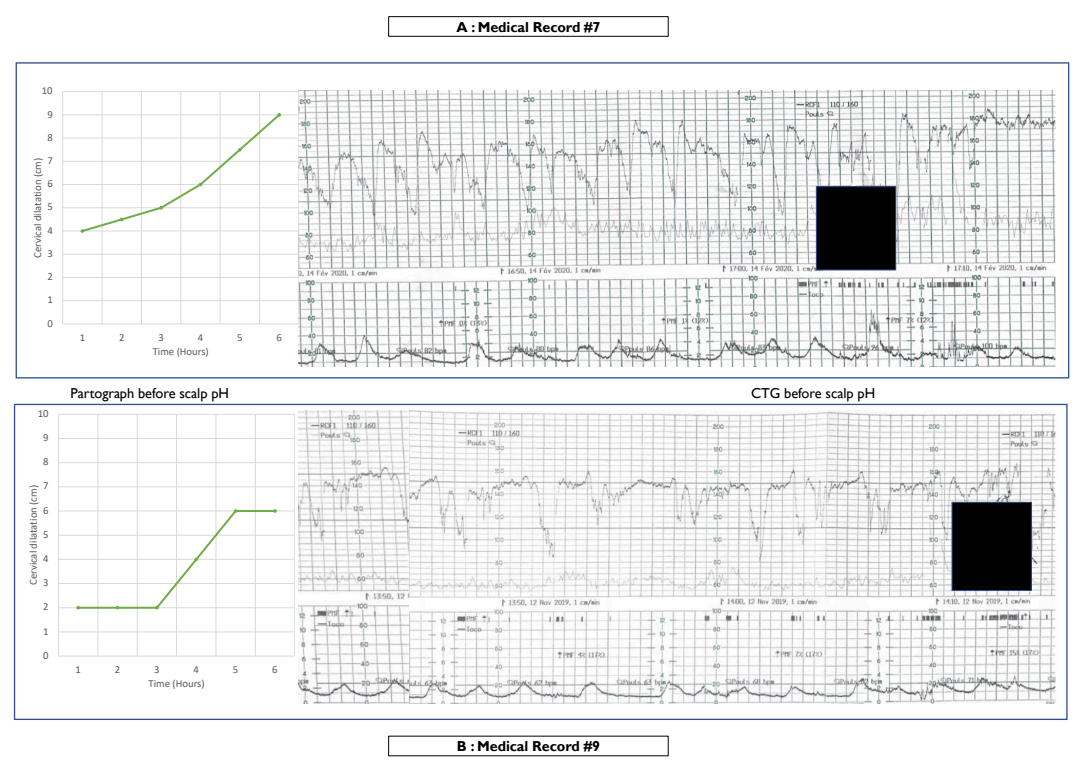

